\title{
Recent advances in the management of lung cancer
}

\author{
Authors: Gavin S Jones ${ }^{\mathrm{A}}$ and David R Baldwin ${ }^{\mathrm{B}, \mathrm{C}}$
}

Historically, the prognosis for individuals diagnosed with lung cancer has been bleak. However, the past 10 years have seen important advances in treatment and diagnosis which have translated into the first improvements seen in lung cancer survival. This review highlights the major advances in treatments with curative intent, systemic targeted therapies, palliative care and early diagnosis in lung cancer. We discuss the pivotal research that underpins these new technologies/strategies and their current position in clinical practice.

KEYWORDS: Lung cancer, curative treatment, targeted therapies, early diagnosis, lung cancer management

\section{Introduction}

For several decades lung cancer has been the most common cancer in the world. In 2014 there were 46,403 new cases of lung cancer diagnosed in the UK. ${ }^{2}$ It is the third most common cancer after breast and prostate, but has the largest proportion of all cancer-related deaths $(22 \%)^{2}{ }^{2}$ The overall age standardised incidence has declined slightly over the past 40 years, which is a combination of a marked decline among men and an increase for women (Fig 1). Approximately $62 \%$ of people have advanced stage disease at diagnosis. ${ }^{3}$ When combining all stages of lung cancer in England, 1-year survival has improved from 24.5\% in 1995-9 to $36.7 \%$ currently. ${ }^{4}$ Much of this improvement has occurred since 2010 and is attributed to developments in lung cancer care.

\section{Staging}

It is important to accurately stage patients with lung cancer as this contributes to treatment options and prognosis. Better access to positron emission tomography with computed tomography (PET-CT) scanning and endobronchial ultrasound (EBUS) for mediastinal lymph node sampling have increased the accuracy of staging for lung cancer.

Authors: ${ }^{\text {A }}$ clinical research fellow, Division of Epidemiology and Public Health, University of Nottingham, Nottingham, UK; ${ }^{B}$ honorary professor of medicine, Division of Epidemiology and Public Health, University of Nottingham, Nottingham, UK; ${ }^{C}$ consultant respiratory physician, Department of Respiratory Medicine, Nottingham University hospitals, Nottingham, UK
The American joint committee on cancer adopted the 8th edition of the International Association for the Study of Lung Cancer's (IASLC) staging project ${ }^{5}$ in January 2017. This replaced the previous edition that was originally published in 2009 . The 8th edition is derived from the IASLC database of 77,156 evaluable cases of histologically confirmed NSCLC diagnosed between 1999 and 2010 from 35 sources in 16 countries. The stage groupings are summarised in Fig 2 and are based on the new TNM ('tumour, nodes and metastases') classification which is as follows.

\section{Tumour}

Primary tumour classification is now subdivided into: $T 1 \mathrm{a} \leq 1 \mathrm{~cm}$, $\mathrm{T} 1 \mathrm{~b}>1$ to $2 \mathrm{~cm}, \mathrm{~T} 1 \mathrm{c}>2$ to $3 \mathrm{~cm}, \mathrm{~T} 2 \mathrm{a}>3$ to $4 \mathrm{~cm}, \mathrm{~T} 2 \mathrm{~b}>4$ to $5 \mathrm{~cm}$, $\mathrm{T} 3>5$ to $7 \mathrm{~cm}$ and $\mathrm{T} 4>7 \mathrm{~cm}$. Tis and T1mi were introduced for adenocarcinoma in situ and minimally invasive adenocarcinoma. Endobronchial tumours located $<2 \mathrm{~cm}$ from the main carina were found to have a better prognosis so have been reclassified as T2 rather than T3. Similarly, total lung atelectasis / pneumonitis is now classed as T2. Diaphragmatic invasion has been upstaged to T4. Mediastinal pleural invasion is no longer used as a descriptor.

Node

Nodal station descriptors have not changed: N1 ipsilateral hilar node, N2 ipsilateral mediastinal or subcarinal node, N3 contralateral mediastinal or supraclavicular/scalene node.

\section{Metastases}

M1a, for intrathoracic metastases, remains unchanged. Extrathoracic metastases have been reclassified into M1b; single extra-thoracic metastasis in a single organ or M1c; multiple extrathoracic metastases in a single organ or multiple organs.

Previously curative treatments were generally reserved for stages I-IIIA; however, the treatment of oligometastatic disease (broadly defined as less than 5 metastases in a single organ ${ }^{6}$ ) is an area of growing research interest. When oligometastatic lung cancer is treated aggressively with ablative radiotherapy or resection, several observational studies have estimated 1-year survival to be $35-56 \% .^{6-8}$ This is a significant improvement if one considers that the overall 1-year survival for people with stage IV lung cancer in the UK is $14 \%{ }^{9}$

\section{Thoracic surgery}

Thoracic surgery is considered the standard of care for people with early stage lung cancer who are deemed fit enough. Modern 
Fig 1. Age standardised ratios of lung cancer in the UK since 1979. Reproduced with permission. Web content: Cancer Research UK, http://www.cancerresearchuk.org/health-professional/ cancer-statistics/statistics-bycancer-type/lung-cancer/incidence ASR $=$ age standardised ratio

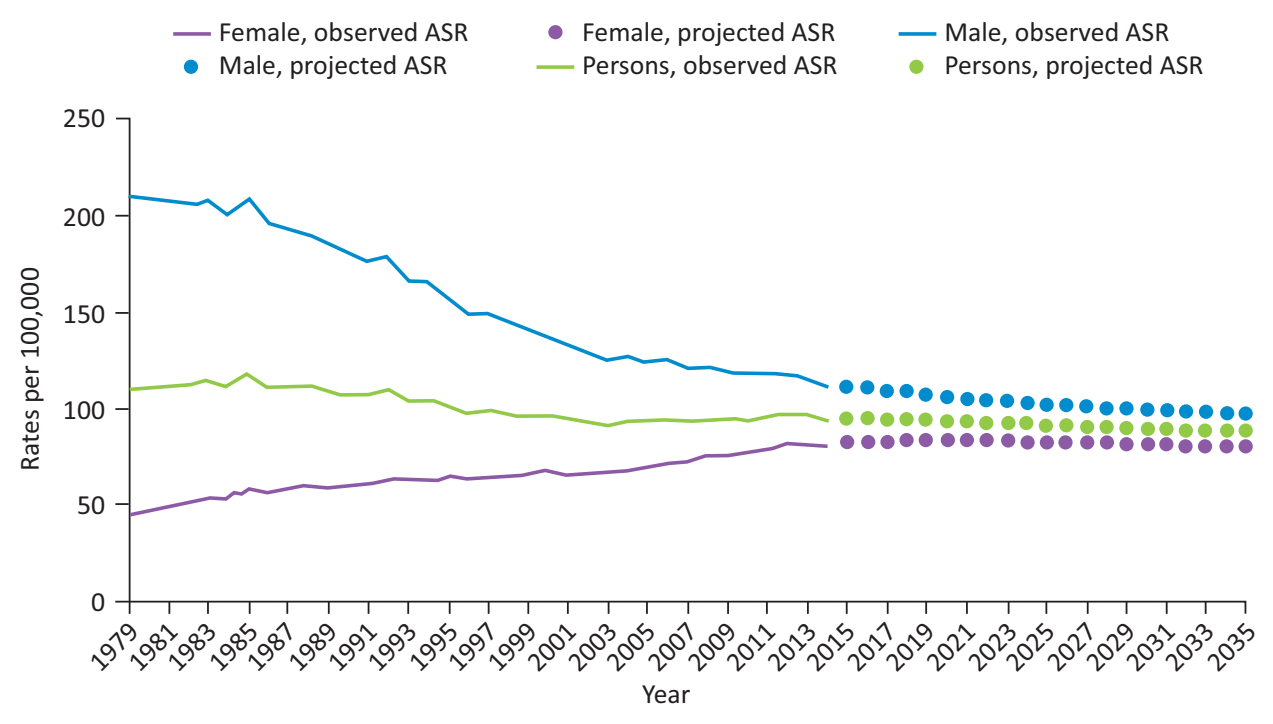

surgical techniques have been developed, including less invasive video-assisted thoracoscopic surgery (VATS) for lung resections (Fig 3), that are changing the boundaries of surgical fitness. Perioperative mortality and long-term survival following VATS lobectomy has been shown to be better than open surgery in some studies. A large European retrospective cohort study found in-hospital mortality following VATS lobectomy to be $1 \%$ vs $1.9 \%$ for open lobectomy. ${ }^{10}$ Similarly, a systematic review and metaanalysis found 5-year survival following VATS lobectomy for early stage lung cancer to be $80.1 \%$ vs $65.6 \%$ for open lobectomy. ${ }^{11}$ Video-assisted thoracoscopic surgery lobectomy also has a lower risk of total complications (29.1\% VATS vs $31.7 \%$ open $^{10}$ ) and a shorter hospital stay ( 8.3 days VATS vs 13.3 days open ${ }^{11}$ ).

Over the past 10 years, surgical resection rates have increased from $9 \%$ to nearly $17 \%^{3}$ and surgeons are now more likely to operate on people who are older than 70 years $^{12}$ (the median age of lung cancer diagnosis is 73 years). The use of lung-sparing surgery has also increased which has resulted in the incidence of pneumonectomy decreasing, an operation which carries an approximate $11 \%$ mortality risk within 90 days. ${ }^{13}$ Laparoscopic

\begin{tabular}{|c|c|c|c|c|c|c|c|}
\hline & NO & N1 & N2 & N3 & M1a & M1b & M1c \\
\hline$T 1 a$ & IA 1 & IIB & IIIA & IIIB & IVA & IVA & IVB \\
\hline$T 1 b$ & IA 2 & IIB & IIIA & IIIB & IVA & IVA & IVB \\
\hline$T 1 c$ & IA 3 & IIB & IIIA & IIIB & IVA & IVA & IVB \\
\hline$T 2 a$ & IB & IIB & IIIA & III & IVA & IVA & IVB \\
\hline$T 2 b$ & IIA & IIB & IIIA & IIIB & IVA & IVA & IVB \\
\hline$T 3$ & IIIB & IIIA & IIIB & IIIC & IVA & IVA & IVB \\
\hline$T 4$ & IIA & IIIA & IIIB & IIIC & IVA & IVA & IVB \\
\hline
\end{tabular}

Fig 2. International Association for the Study of Lung Cancer (IASLC) 8th edition lung cancer stage groupings. International Association for the Study of Lung cancer 2015. robotic surgery is being developed but it is not currently recommended in the UK.

There are inequalities in access to thoracic surgery across the UK. The 2016 National Lung Cancer Audit reported that the range of patients with non-small cell lung cancer (NSCLC) who received surgical treatment varied from $5 \%$ to $36 \%$ across different trusts. ${ }^{3}$ Previous studies have also demonstrated that a person can be up to $51 \%$ more likely to receive thoracic surgery for lung cancer if they are first diagnosed in a thoracic surgical centre, ${ }^{14,15}$ and this effect is most pronounced in surgical centres with the largest lung cancer populations. ${ }^{15}$

\section{Radical radiotherapy}

Radiotherapy continues to evolve and there are different techniques now being used to treat lung cancer with curative

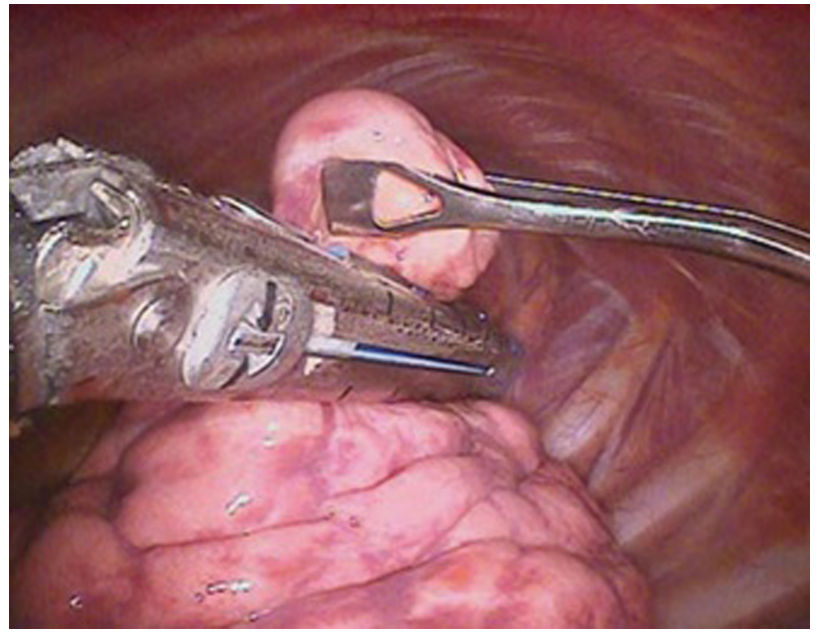

Fig 3. Video-assisted thoracoscopic surgery (VATS). Reproduced with permission of the $\odot$ ERS 2018: The European Lung White Book Respiratory Health and Disease in Europe, 2nd Ed. European Respiratory Society, Sheffield, UK, 2013. Print ISBN: 978-1-84984-042-2, Online ISBN: 978-1-84984-043-9. 


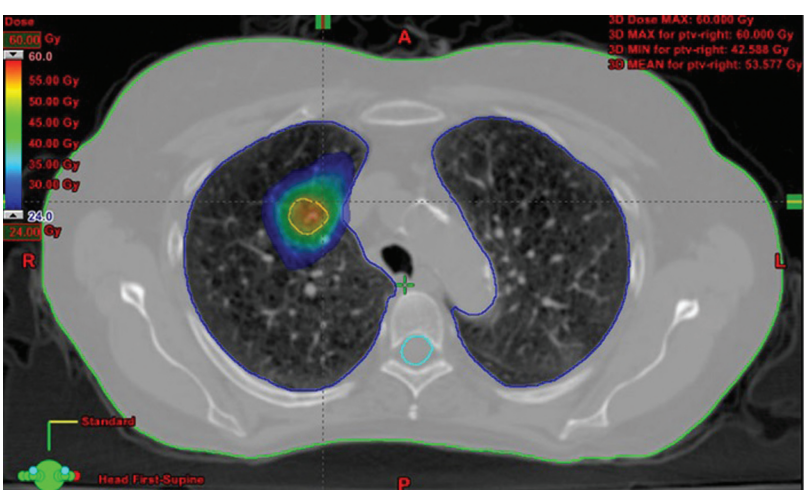

Fig 4. A planning CT thorax for Stereotactic Ablative Radiotherapy (SABR). Reproduced and adapted with permission from Frontiers in oncology: www.frontiersin.org/articles/10.3389/fonc.2015.00213/full $\mathrm{CT}=$ computed tomography

intent. Stereotactic ablative radiotherapy (SABR), which has been developed for use in lung cancer since the early 2000s, but not widely used in the UK until the late 2000s, is able to deliver large doses of radiation with a high precision of $1-2 \mathrm{~mm}$ to small lesions of $<1 \mathrm{~cm}^{3}$ using an external 3D coordinated system that is linked with movements during the respiratory cycle (Fig 4). It has primarily been reserved for people with early stage cancer who have been unable/unwilling to undergo surgical resection due to medical comorbidities. A meta-analysis of observational studies has demonstrated that SABR has a survival benefit over conventional curative intent radiotherapy (2-year survival 70\% with SABR vs $53 \%$ for conventional radiotherapy). ${ }^{16}$ A phase II prospective cohort study also found 3-year survival to be $55.8 \%$ with SABR in T1-2 N0 M0 lung cancers. ${ }^{17}$ As well as improved overall survival, SABR also has better rates of local disease control compared to conventional radiotherapy at 3 years ( $87.2 \%$ SABR vs $43 \%$ conventional radiotherapy). ${ }^{17,18}$

Given its success in treating inoperable patients, research is now focused on its use in patients who would be medically fit for surgery. Research evidence has so far been restricted to propensity matching and the two best studies show conflicting results, one favouring $\mathrm{SABR}^{19}$ and the other surgery for early stage lung cancer. ${ }^{20}$ There have been attempts to run randomised controlled trials but these have failed to recruit adequately. Three further trials are underway.

\section{Radiofrequency / microwave ablation}

Originally used for the treatment of primary or secondary hepatic tumours, percutaneous radiofrequency ablation (RFA) for lung tumours was first described in 2000 by Dupuy et al. ${ }^{21}$ It is used for early stage peripherally based lung tumours or metastases in medically inoperable patients. Under CT guidance, an expandable needle which contains multiple electrodes is inserted percutaneously into the lung lesion (Fig 5). A sinusoidal current is then passed through the electrodes causing thermal destruction of cells and coagulation necrosis. Alternatively, a microwave probe can be used to achieve the same ablative effect. The commonest complication reported is pneumothorax; however, only $4-16 \%$ of patients require chest drain insertion. ${ }^{22}$ There are no studies comparing outcomes from RFA with surgical resection; although, cases series report 75\% overall survival in stage I inoperable lung cancer. ${ }^{23}$

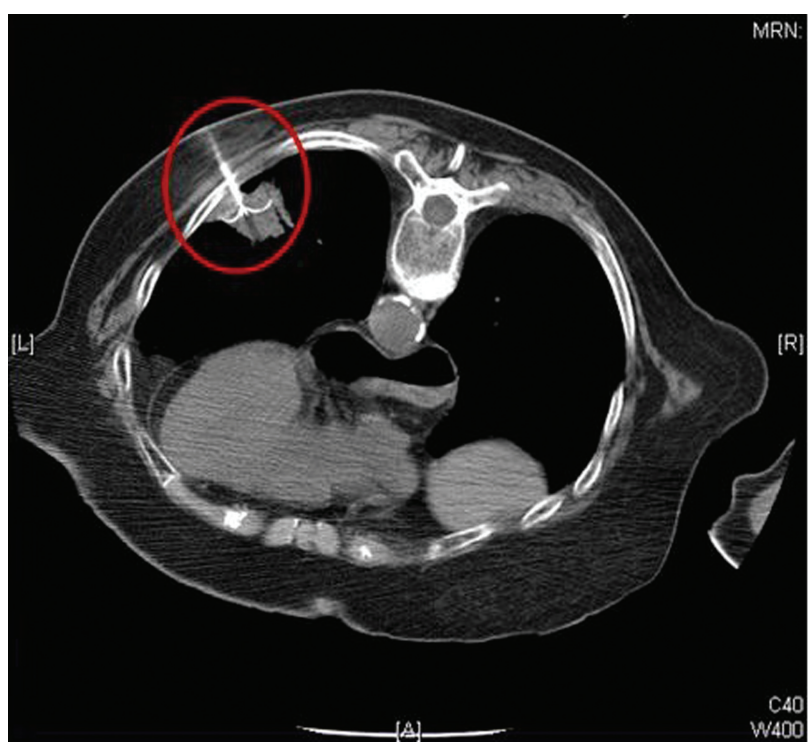

Fig 5. CT scan demonstrating multiple radiofrequency ablation probes being deployed within a peripheral tumour. Reproduced with permission from the Global Resource for Advancing Cancer Education (www.cancerGRACE.org) CT = computed tomography

\section{Systemic therapies}

In contrast to treatments for small cell, chemotherapy for NSCLC has revolutionised over recent years and is becoming increasingly more targeted and individually tailored to each patient based on identification of driver genetic mutations; epidermal growth factor receptor (EGFR), epidermal growth factor receptor thr790met (EGFR T790M), anaplastic lymphoma kinase (ALK) and ROS proto oncogene 1 (ROS-1). It has therefore become more important than ever to obtain a histological diagnosis in those physically fit enough to have treatment, particularly as these newer treatments are better tolerated by patients than standard chemotherapy.

The notion of targeted treatments based on lung cancer histological subtype first became apparent as people with adenocarcinoma were found to have superior survival with cisplatin/pemetrexed chemotherapy compared to cisplatin/ gemcitabine, whereas the opposite was true for those with squamous cell histological type. ${ }^{24}$

The first genetically targeted treatment for NSCLC was gefitinib. This is an orally administered treatment which effects the EGFR through tyrosine kinase inhibition. The EGFR is a transmembrane surface protein that is activated by the binding of epidermal growth factor. Once bound, an intracellular tyrosine kinase domain causes a cascade of events, primarily DNA synthesis and cellular proliferation. In approximately $15 \%$ of people with NSCLC, control of the tyrosine kinase domain in the EGFR is lost due to a mutation in the EGFR gene leading to uncontrolled proliferation. When initially given to all patients with NSCLC, gefitinib was found to have a response in a certain subgroup of patients, predominantly Asian, female, adenocarcinomas and never-smokers. ${ }^{25}$ This prompted researchers to investigate for mutations in the EGFR gene which proved to be overexpressed in those who responded to gefitinib. ${ }^{26} \mathrm{~A}$ further randomised control trial by Maemondo et al was pivotal as all patients with NSCLC harbouring EGFR mutations were randomised to receive either gefitinib or standard 
chemotherapy (paclitaxel and carboplatin). The gefitinib group had a significantly longer progression free survival of 10.8 months vs 6.4 months in the chemotherapy group. ${ }^{27}$ There are now several approved treatments by The National Institute for Health and Care Excellence (NICE) for use in people with EGFR mutation positive NSCLC: erlotinib, afatanib and gefitinib. Other targeted treatments which have been approved by NICE are crizotinib for ALK / ROS-1 mutation positive NSCLC and osimertinib for EGFR T790M mutation positive NSCLC.

The newest class of systemic treatments are immune checkpoint inhibitors: pembrolizumab, nivolumab and atezolizumab. They act through the programmed death-ligand 1/2 (PD-L1 and PD-L2) and programmed death 1 (PD-1) receptor pathway. PD-L1 and PD-L2 are proteins which are thought to suppress the immune system by binding with the PD-1 receptor on activated T cells (which are responsible for causing cytotoxic death of cancer cells when activated by tumour antigens). Some cancer cells have been found to express PD-L1 and PD-L2 on their cell membrane, effectively providing a cloak of protection from the immune system. These immunotherapies block the PD-L1/2 and PD-1 receptor pathway, thereby removing the brakes on the immune system, allowing cancer cells to be identified and undergo cytotoxic $T$ cell mediated death. PD-L1 testing was developed as part of the KEYNOTE-001 trial and is carried out via immunohistochemistry staining of tumour cells and calculating the proportion of cells stained. The KEYNOTE-010 trial demonstrated that pembrolizumab at two different doses significantly improved overall survival compared to standard chemotherapy (docetaxel) in people with previously treated NSCLC who had $>1 \%$ expression of PD-L1 tumour cells. ${ }^{28}$ Similar findings have also been found with nivolumab compared to docetaxel in patients with $>1 \%$ PD-L1 expression. ${ }^{29}$ CTLA-4 (cytotoxic T-lymphocyte-associated protein 4 ) is another molecule present on lymphocytes that down regulates the immune system for which inhibitors are available. Ipililumab, a CTLA-4 inhibitor, has shown promising results ${ }^{30}$ and phase III trials of this drug in combination with other systemic anticancer therapies to treat NSCLC, such as the checkmate 227 trial, are currently in progress.

These treatments are expensive and it is estimated the cost per quality-adjusted life year (QALY) of pembrolizumab is approximately $£ 50,000$. Despite its significant cost, pembrolizumab has been approved by NICE for use within the cancer drugs fund, as first-line treatment for PDL-1 positive $>50 \%$ tumour staining) advanced stage NSCLC. Similarly, nivolumab as well as pembrolizumab have both been recommended by NICE to treat previously treated advanced stage PDL-1 positive NSCLC.

\section{Supportive and palliative care}

Specialist palliative care also has a vital role in lung cancer care and much work has been carried out to optimise its use and improve patient outcomes. Temel et al examined the effect of early specialist palliative care support compared with standard care in ambulatory patients with metastatic NSCLC referred to the medical oncology outpatient department. ${ }^{31}$ They found a significant difference in median survival with the early supportive care group, 11.6 months, compared to 8.9 months in the standard care group. These patients also had a better quality of life score and fewer depressive symptoms (based on questionnaire results) and were less likely to require aggressive end-of-life care support. The finding is supported by another trial, which found an improvement in 1 -year survival in patients who received early supportive care. ${ }^{32}$
Integrating early enhanced supportive care for people with advanced cancer into standard oncology care has been recommended by the American Society of Clinical Oncology, which, after conducting a systematic review of clinical trials concluded there is strong evidence that early palliative care improves quality of life, reduces depression and improves satisfaction with care; however, there was less evidence to show that it improves survival. ${ }^{33}$ Integrated palliative care and oncology services vary by hospital in the UK as no national guidance exists. This integration is arguably a more cost-efficient use of resources than approving use of some of the systemic targeted treatments as not a single trial of enhanced supportive care has demonstrated a cost increase over routine care. ${ }^{33}$

\section{Early detection}

A significant shift in the outcomes of lung cancer may lie with improved early detection. Broadly, this can be augmented by introducing screening initiatives, improving awareness and recognition of lung cancer and clearer referral pathways.

Screening for lung cancer using low dose CT scanning is currently under consideration by the UK National Screening Committee. It has been shown as an effective way to detect early stage lung cancers and improve mortality compared to chest X-ray. The largest trial, the National Lung Cancer Screening Trial (NLST), ${ }^{34}$ was conducted in the USA. It randomised 53,454 people who were 55-74 years old and were current or ex-smokers in the past 15 years, with a 30 or more pack year history, to receive either a CT chest or plain chest X-ray. The authors found a $20 \%$ reduction in lung cancer specific mortality rate and a reduction of $6.7 \%$ in overall mortality rate in the CT group. A similar pilot trial has been conducted in the UK, the UK Lung Cancer Screening Trial (UKLS), ${ }^{35}$ and has used a more selective method of identifying patients for CT screening by sending a questionnaire to identify high-risk individuals (defined as $\geq 5 \%$ 5-year lung cancer risk) using the Liverpool Lung Project risk score. Those identified were then randomised to $\mathrm{CT}$ or no intervention. The lung cancer detection rate was $2.1 \%$, the majority of which $(86 \%)$ were stage I and II. The resection rate was $83 \%$ with $10 \%$ of resections being done for benign disease (less than half that in other screening trials). The incremental cost-effectiveness ratio was estimated to be $£ 8,466$ ( $£ 5,542-£ 12,569$ ) per QALY. Other European trials have been conducted with mixed results, partly due to inadequate study design. The Dutch-Belgian trial, NELSON, is due to report on mortality outcomes in the near future and the results may influence the decision of whether to implement lung cancer screening in the UK.

Before screening can be implemented many questions remain and much research has been focused to address the concerns of cost effectiveness, screening intervals, selection criteria, participation rates, optimal diagnostic workup and minimising harm as well as incorporating effective smoking cessation. Although there is no national screening programme there have been many localised CT screening pilots set up throughout the UK as part of the 'Accelerate, coordinate and evaluate' project by Cancer Research UK and through other funding sources, these are still ongoing.

Increasing awareness through national media campaigns such as 'Be Clear on Cancer', implemented in 2012, have been used to augment early diagnosis. When evaluated the campaign was estimated to have led to 700 additional lung cancers being diagnosed compared to the previous year, with approximately 400 more people having an earlier stage at diagnosis. ${ }^{36}$ Improving 


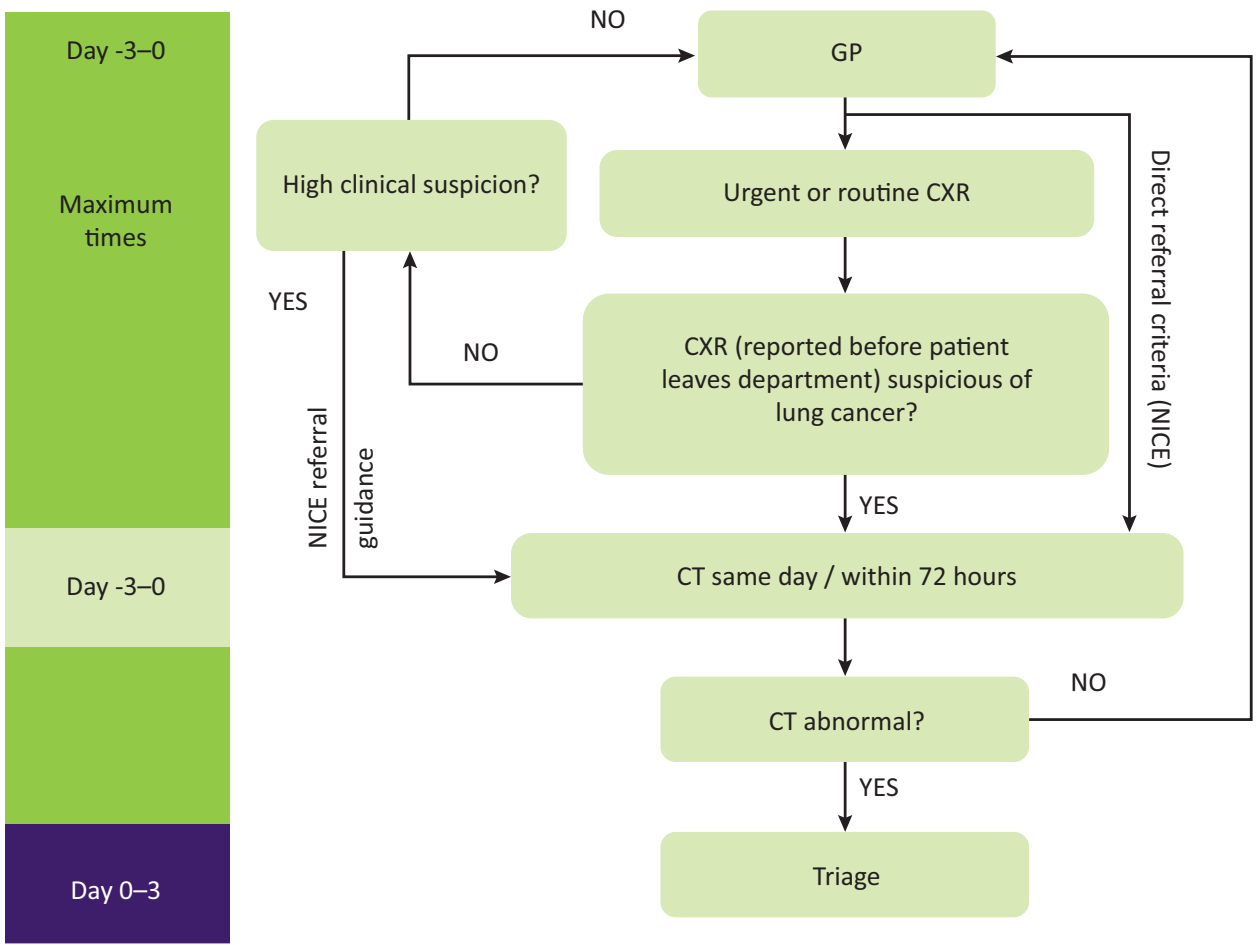

Fig 6. First stage of the optimum lung cancer pathway. 0 on the maximum waiting times bar corresponds to the date of referral onto the cancer pathway. $\mathrm{CRX}=$ chest $\mathrm{X}$-ray; $\mathrm{CT}=$ computed tomography; NICE = National Institute for Health and Care Excellence awareness among healthcare professionals as well as patients is also needed. Research has suggested that people who die within 90 days of a lung cancer diagnosis have more interactions with their GP prior to diagnosis than those who lived longer, suggesting that earlier opportunities to capture the diagnosis are being missed perhaps due to lack of awareness. ${ }^{37}$

Augmenting early detection also requires clear and efficient referral pathways. The Clinical Expert Group for lung cancer, NHS England, has produced guidance to assist commissioners in allocating resources to balance the hub and spoke inequalities in lung cancer care. It has also developed a National Optimum Lung Cancer Pathway. Part of this new pathway recommends that the chest $\mathrm{X}$-ray is reported while the patient is within the radiology department and if it is abnormal the patient undergoes a CT chest the same day or within 72 hours (Fig 6). The new pathway would also allow primary care direct access to CT scanning. These changes could reduce referral times by weeks as they bypass the inherent delays of the report reaching the GP and the subsequent GP referral / further secondary care CT scan. This may also decrease emergency presentations as patients are captured earlier. In order to best select patients for referral from primary care several lung cancer risk scores have been developed; however, they all need to be compared head-to-head to assess which performs best so that radiology services are not overwhelmed by chest $\mathrm{X}$-ray and $\mathrm{CT}$ scan requests once the pathway is introduced.

\section{Conclusion}

Many technical, pharmacological and service developments have been made in the staging and treatment of lung cancer over the past 10 years but questions still remain about how to best implement these and their cost effectiveness. Further research is needed to ascertain whether newer radiotherapy techniques, such as SABR, are equivalent to surgery for early stage lung cancers. Much discussion still surrounds the newer targeted agents' cost effectiveness and whether improving early supportive care might be a good use of resources.

Although new treatments are available there are inequalities in access to them and further consideration in commissioning of resources is needed to tackle the hub and spoke effect. Arguably the most effective development that has been made in improving the outcomes for lung cancer is CT screening; however, it still remains to be introduced in the UK despite good evidence for effectiveness.

\section{Conflicts of interest}

DRB has received an educational grant to support the Cambridge chest meeting from AGFA, Boehringer Ingelheim, Irwin Mitchell and Roche. Travel support was received from Oncimmune Ltd.

GSJ's current employment is funded by Roy Castle Lung Cancer foundation and University of Nottingham. He previously received a travel grant from Actelion Pharmaceuticals to attend a pulmonary hypertension preceptorship.

\section{References}

1 International Agency for Research on Cancer. globocan 2012: Estimated cancer incidence, mortality and prevalence worldwide 2012. http://globocan.iarc.fr/Pages/fact_sheets_cancer.aspx [Accessed 24 February 2016].

2 Cancer research UK. Lung cancer statistics www.cancerresearchuk. org/health-professional/cancer-statistics/statistics-by-cancer-type/ lung-cancer [Accessed 24 February 2016].

3 Royal College of Physicians. The National Lung Cancer Audit report 2016. London: RCP, 2016. 
4 Walters S, Benitez-Majano S, Muller P et al. Is England closing the international gap in cancer survival? Br J Cancer 2015;113:848-60.

5 Goldstraw P, Chansky K, Crowley J et al. The IASLC Lung Cancer Staging Project: Proposals for Revision of the TNM Stage Groupings in the Forthcoming (Eighth) Edition of the TNM Classification for Lung Cancer. J Thorac Oncol;11:39-51.

6 De Ruysscher D, Wanders R, van Baardwijk A et al. Radical treatment of non-small-cell lung cancer patients with synchronous oligometastases: long-term results of a prospective phase II trial (Nct01282450). J Thorac Oncol 2012;7:1547-55.

7 Tanvetyanon T, Robinson LA, Schell MJ et al. Outcomes of adrenalectomy for isolated synchronous versus metachronous adrenal metastases in non-small-cell lung cancer: a systematic review and pooled analysis. J Clin Oncol 2008;26:1142-7.

8 Moreno P, de la Quintana Basarrate A, Musholt T] et al. Adrenalectomy for solid tumor metastases: results of a multicenter European study. Surgery 2013;154:1215-22; discussion 1222-3.

9 Office for National Statistics. Cancer survival by stage at diagnosis for England (experimental statistics): Adults diagnosed 2012, 2013 and 2014 and followed up to 2015. ONS, 2016.

10 Falcoz PE, Puyraveau M, Thomas PA et al. Video-assisted thoracoscopic surgery versus open lobectomy for primary non-smallcell lung cancer: a propensity-matched analysis of outcome from the European Society of Thoracic Surgeon database. Eur ] Cardiothorac Surg 2016;49:602-9.

11 Whitson BA, Groth SS, Duval S], Swanson S], Maddaus MA. Surgery for early-stage non-small cell lung cancer: a systematic review of the video-assisted thoracoscopic surgery versus thoracotomy approaches to lobectomy. Ann Thorac Surg 2008;86:2008-16; discussion 16-8.

12 Riaz SP, Linklater KM, Page $\mathrm{R}$ et al. Recent trends in resection rates among non-small cell lung cancer patients in England. Thorax 2012;67:811-14

13 Powell HA, Tata LJ, Baldwin DR et al. Early mortality after surgical resection for lung cancer: an analysis of the English National Lung cancer audit. Thorax 2013;68:826-34.

14 Rich AL, Tata LJ, Free CM et al. Inequalities in outcomes for nonsmall cell lung cancer: the influence of clinical characteristics and features of the local lung cancer service. Thorax 2011;66:1078-84.

15 Khakwani A, Rich AL, Powell HA et al. The impact of the "hub and spoke' model of care for lung cancer and equitable access to surgery. Thorax 2015;70:146-51.

16 Grutters JP, Kessels AG, Pijls-Johannesma M et al. Comparison of the effectiveness of radiotherapy with photons, protons and carbon-ions for non-small cell lung cancer: a meta-analysis. Radiother Oncol 2010;95:32-40.

17 Timmerman R, Paulus R, Galvin ] et al. Stereotactic body radiation therapy for inoperable early stage lung cancer. JAMA 2010;303:1070-6.

18 Lagerwaard FJ, Senan S, van Meerbeeck JP et al. Has 3-D conformal radiotherapy (3D CRT) improved the local tumour control for stage I non-small cell lung cancer? Radiother Oncol 2002;63:151-7.

19 Verstegen NE, Oosterhuis JW, Palma DA et al. Stage I-II nonsmall-cell lung cancer treated using either stereotactic ablative radiotherapy (SABR) or lobectomy by video-assisted thoracoscopic surgery (VATS): outcomes of a propensity score-matched analysis. Ann Oncol 2013:24:1543-8.

20 Paul S, Lee PC, Mao J, Isaacs AJ, Sedrakyan A. Long term survival with stereotactic ablative radiotherapy (SABR) versus thoracoscopic sublobar lung resection in elderly people: national population based study with propensity matched comparative analysis. BMJ 2016;354:i3570.

21 Dupuy DE, Zagoria RJ, Akerley W et al. Percutaneous radiofrequency ablation of malignancies in the lung. AJR Am J Roentgenol 2000;174:57-9.
22 Bargellini I, Bozzi E, Cioni R, Parentini B, Bartolozzi C. Radiofrequency ablation of lung tumours. Insights into Imaging 2011:2:567-76.

23 Lencioni R, Crocetti L, Cioni R et al. Response to radiofrequency ablation of pulmonary tumours: a prospective, intention-to-treat, multicentre clinical trial (the RAPTURE study). Lancet Oncol 2008;9:621-8

24 Scagliotti GV, Parikh P, von Pawel ] et al. Phase III study comparing cisplatin plus gemcitabine with cisplatin plus pemetrexed in chemotherapy-naive patients with advanced-stage non-small-cell lung cancer. J Clin Oncol 2008:26:3543-51.

25 Kris MG, Natale RB, Herbst RS et al. Efficacy of gefitinib, an inhibitor of the epidermal growth factor receptor tyrosine kinase, in symptomatic patients with non-small cell lung cancer: A randomized trial. JAMA 2003;290:2149-58.

26 Lynch T], Bell DW, Sordella R et al. Activating mutations in the epidermal growth factor receptor underlying responsiveness of nonsmall-cell lung cancer to gefitinib. N Engl ] Med 2004;350:212939.

27 Maemondo M, Inoue A, Kobayashi K et al. Gefitinib or chemotherapy for non-small-cell lung cancer with mutated EGFR. N Engl ] Med 2010;362:2380-88.

28 Herbst RS, Baas P, Kim DW. Pembrolizumab versus docetaxel for previously treated, PD-L1-positive, advanced non-small-cell lung cancer (KEYNOTE-010): a randomised controlled trial. Lancet 2016;387:1540-50.

29 Borghaei $\mathrm{H}$, Paz-Ares L, Horn L et al. Nivolumab versus Docetaxel in Advanced Nonsquamous Non-Small-Cell Lung Cancer. N Engl J Med 2015:373:1627-39.

30 Antonia S], López-Martin JA, Bendell J et al. Nivolumab alone and nivolumab plus ipilimumab in recurrent small-cell lung cancer (CheckMate 032): a multicentre, open-label, phase 1/2 trial. Lancet Oncol 2016;17:883-95.

31 Temel JS, Greer JA, Muzikansky A et al. Early Palliative Care for Patients with Metastatic Non-Small-Cell Lung Cancer. N Engl J Med 2010;363:733-42.

32 Bakitas MA, Tosteson TD, Li Z et al. Early Versus Delayed Initiation of Concurrent Palliative Oncology Care: Patient Outcomes in the ENABLE III Randomized Controlled Trial. J Clin Oncol 2015;33:1438-45

33 Ferrell BR, Temel JS, Temin S et al. Integration of Palliative Care Into Standard Oncology Care: American Society of Clinical Oncology Clinical Practice Guideline Update. J Clin Oncol 2017:35:96-112.

34 National Lung Screening Trial Research Team, Aberle DR, Adams AM et al. Reduced lung-cancer mortality with low-dose computed tomographic screening. N Engl J Med 2011;365:395-409.

35 Field JK, Duffy SW, Baldwin DR et al. UK Lung Cancer RCT Pilot Screening Trial: baseline findings from the screening arm provide evidence for the potential implementation of lung cancer screening. Thorax 2016;71:161-70.

36 Cancer Research UK. Be Clear on Cancer evaluation update. CRUK 2014.

37 O'Dowd EL, McKeever TM, Baldwin DR et al. What characteristics of primary care and patients are associated with early death in patients with lung cancer in the UK? Thorax 2015;70:161-8.

Address for correspondence: Prof David Baldwin, Department of Respiratory Medicine, David Evans Building, Nottingham University Hospitals, City campus, Nottingham NG5 1PB, UK. Email: David.Baldwin@nottingham.ac.uk 